\title{
Communicating a Sales Job to Occupational Changers: A Qualitative Content Analysis of Online Job Advertisements
}

\author{
Gerriet Hinrichs ${ }^{1}$, Henning Bundtzen ${ }^{1}$ \\ ${ }^{1}$ Szent-István University, Kaposvár, Hungary
}

\begin{abstract}
This paper analyses insurance companies' online communication to potential occupational changers into sales by a qualitative content analysis of corresponding online job advertisements. Since the addressees do not know this job in detail, a realistic job preview was expected. In the structuring part of the content analysis, the main categories of the online job advertisements were determined first, after which the summarizing part shows the contents in a first and second reduction. It turns out that neither the tasks nor the requirements or compensations are described sufficiently to enable potential candidates to assess their interest or suitability.
\end{abstract}

Keywords - Online Job Advertisements, Occupational Change, Recruiting.

\section{Introduction}

When people outside the insurance sales think of the profession of insurance agent, they often envision a type like Gil Gundersun from The Simpsons. [1] explored common stereotypes and prejudices about salespersons by using an association technique and qualitative analysis to create an inventory of most associated characteristics of salespeople.

DOI: 10.18421/TEM102-45

https://doi.org/10.18421/TEM102-45

Corresponding author: Gerriet Hinrichs, Szent-István University, Kaposvár, Hungary. Email: gerriethinrichs@outlook.com

Received: 10 February 2021.

Revised: 15 April 2021.

Accepted: 20 April 2021.

Published: 27 May 2021.

(cc) BY-NC-ND (C) 2021 Gerriet Hinrichs \& Henning Bundtzen; published by UIKTEN. This work is licensed under the Creative Commons Attribution-NonCommercial-NoDerivs 4.0 License.

The article is published with Open Access at www.temjournal.com
18 out of 29 identified characteristics were negatively connoted. The typical salesperson is found in consistently average characteristics, while customer-focused, well-educated, trustworthy and a not expensive but stylish suit turn out to be atypical attributes. Noteworthy here is the fact that knowledge about product and customer-orientation are the only ones of the 29 attributes that are related to the job. Words such as structured, hard-working, or performance-related income do not appear here. This illustrates how little is known about the sales job outside the industry. However, if occupational changers are to be addressed in order to fill vacant sales jobs, these negative connotations should be eliminated. Realistic Job Previews (RJP) offers a possibility for this.

In a labor market, employers and employees face each other with incomplete information. In order to overcome this asymmetry, they send out appropriate signals of suitability according to Signaling Theory [2]. Since employers want to gain a competitive advantage through the best employees, they send persuasive signals under the term "recruitment" to attract applicants and influence job choice [3]. RJP is one of the three most intensively studied areas in recruitment research [4]. RJP are based on the met expectations hypothesis, according to which positive outcomes of an occupational choice are more likely to occur if the expectations of an occupation or job are fulfilled to a sufficient degree. An older but not outdated meta-analysis on this is offered by [5]. Clear positive correlations are found here between met expectations and job satisfactions as well as organizational commitment. The reason for this is seen in the lowering of unrealistically high expectations when all relevant information, both positive and negative, is available to the candidate in a balanced manner. [6] found the primary mechanisms of the frequently shown negative RJPturnover correlation also in met expectations, but further in role clarity, self-selection, and perceptions of fit and the organizations' honesty.

[7] analyzed the issue of self-selection. While the amount and type of information had a significant 
positive influence on the attractiveness of a job, they showed that the amount of negative information could lead to the problem of adverse self-selection with a significant negative and superior coefficient. This problem, that basically desirable contractors refrain from cooperation due to sufficient information asymmetries, arises in several contexts, such as the famous market for lemons [8]. Since no relevance for the attractiveness of the job was found in the source of negative information, they conclude that indeed only the pure quantity seems to be decisive. [9], in distinguishing between quality and quantity of information at the hiring stage, do not focus on the attractiveness before starting the job but on the propensity to leave after at least one year of experience being a salesperson. In their study, it is in particular the items relevance and depth of information that are rated significantly lower by those with an intention to leave, while quantity is also rated lower but to a lesser extent. [10] emphasize as a result of their meta-analysis that RJP primarily positively influence the perceptions of honesty of the company providing information and thus the attractiveness of the company. Furthermore, they demonstrate a positive effect of this honesty on retention. Of course, this may also be due to the fact that there are indeed differences in the credibility of recruiters and companies. The medium of the information offered also has a high value. For example, verbal RJP offer scope for individual inquiries and an assessment of the credibility of the source due to the high degree of interaction. With written RJP, on the other hand, the advantage lies in the effectiveness and degree of standardization of the information [10]. It is also easy to comprehend that a pre-hire RJP has a stronger effect than a post-hire one.

The initial source of information for a candidate is the job advertisement. Accordingly, it makes sense to analyze the content of the information offered therein.

\section{Materials and Methods}

We examined the online job advertisements of the 25 largest German non-life insurance companies by gross premiums earned according to BaFin's primary insurer statistics [11] with own sales force. These companies represent over $75 \%$ of the total gross premiums earned in Germany in 2019. Non-life insurance companies were preferred to life insurance companies as they are likely to be significantly more noticeable by customers and potential candidates due to more frequent contact (higher claims frequency, need for adjustment and change in preference). Corporations with multiple companies under the same brand were grouped into one company. First, it was investigated whether a company offers the possibility of a lateral entry into sales in the form of an online job advertisement on the company's website. If this was the case, the job advertisement was exported as a PDF and loaded into the MAXQDA software. If this was not directly apparent, job ads were then searched for on indeed.de with the string "*NAME OF COMPANY* Vertrieb" without any location limitation. If the possibility of an occupational change into sales was found there in the form of an online job advertisement, the job advertisement was also integrated into the sample. This resulted in 18 job ads for occupational changers into insurance sales to be evaluated. $72 \%$ of the analyzed companies offered this possibility. While $41.6 \%$ of the 12 mutual insurance companies in the data set did not accept occupational changers, only one of the 10 stock companies was not willing to do so. The analysis was carried out according to the qualitative content analysis method proposed by [12]. In the first step of a structuring content analysis, the main categories "Tasks/Responsibilities", "Requirements/Qualifications" and "Compensation/Benefits" were applied to the material and supplemented. After this principally deductive categorization, a summary content analysis of inductive form took place for each main category according to the process model of paraphrasing, generalization, first reduction and second reduction. On this basis, the core statements for each main category were then identified.

\section{Results}

The structuring content analysis revealed the following basic structure in the online job advertisements examined (anchor examples in brackets):

(1) Job Title (Heading, bold);

(2) Tasks / Responsibilities

("Your responsibilities", "Your challenges");

(3) Requirements / Qualifications ("Your Profile", "What you can provide");

(4) Compensation / Benefits

("What we can offer you ", "What you can expect of us");

(5) Introduction / Frame

(Presentation of the company: history, culture, size);

In the following, the results of the summarizing content analysis are listed individually for each main category. Both the first and, except for the Job Title, the second reduction are shown. The number of job ads that contain a statement according to the respective code is also given. Since the aim is to make a generalizable statement about the communicated occupation of insurance agent, only 
the 10 most frequently mentioned codes per main category are examined in more detail. For the same reason, the authors refrain from evaluating the Introduction/Frame, which does not contain any statements about the occupation as such.

The first reduction of the Job Titles results in dominance of the categories "Sales Representative" and "Customer Advisor" are shown in Figure 1. Since some titles could be assigned to more than one code, this leads to a larger number of codes than job titles.

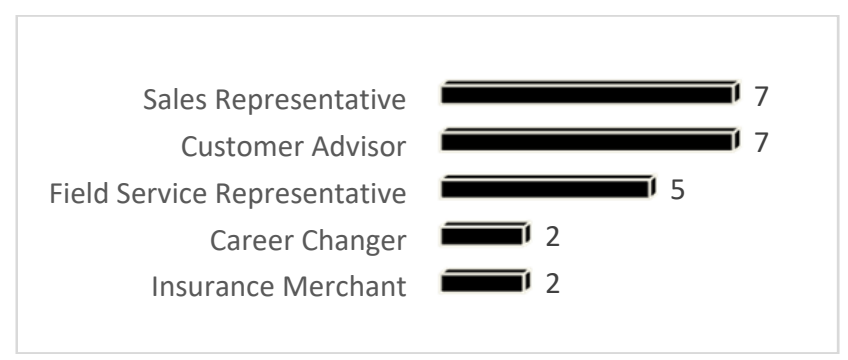

Figure 1. First Reduction Job Titles

The representation of the Job Tasks of the proposed job covers on average $10 \%$ of the online job advertisement with a range of $1 \%-31 \%$. Figure 2 gives an overview of the 10 most frequently mentioned first reductions.

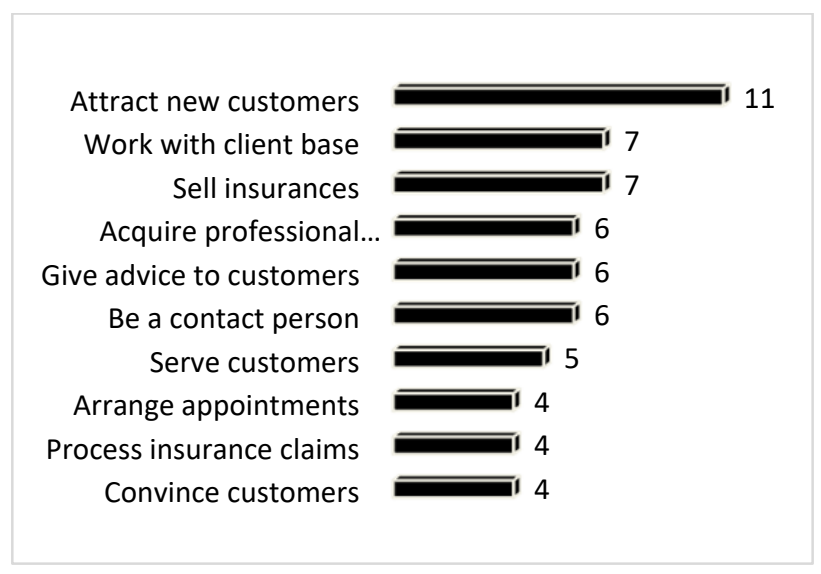

Figure 2. First Reduction Job Tasks

On average, $12 \%$ of the job ad is spent on describing the Job Requirements, with a range of $2 \%$ - $18 \%$. An overview is given in Figure 3.

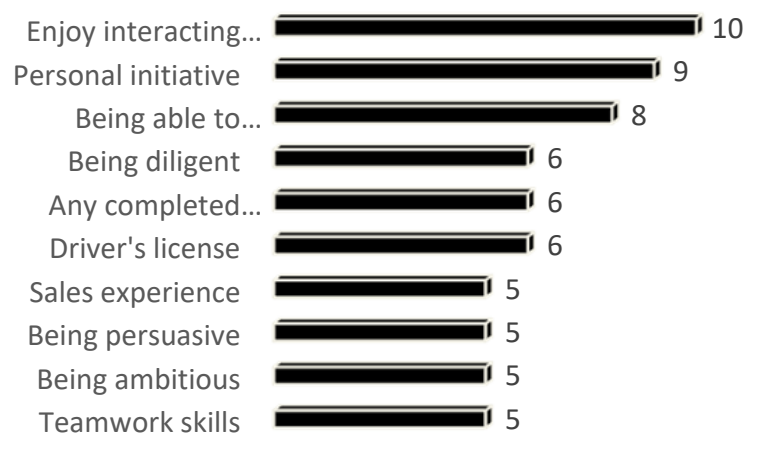

Figure 3. First Reduction Job Requirements
Employers also use an average of $12 \%$ of the available space for the benefits gained by choosing this job or occupation. Here the range is between $2 \%$ and 38\%. Figure 4 shows the benefits offered in descending order of nomination.

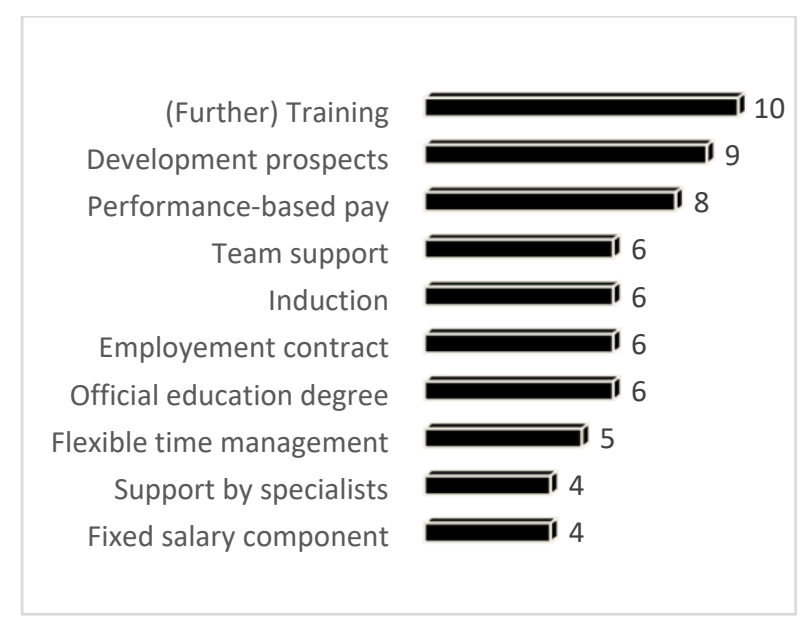

Figure 4. First Reduction Job Compensation

Table 1 provides an overview of the number of codes grouped under Second Reduction in all online job advertisements. It also shows the average and median number of mentions in this category per job ad and the number of job ads without a mention in this category.

Table 1. Second Reduction of Main Categories

\begin{tabular}{|l|c|c|c|c|}
\hline 2nd Reduction & Count & Average & Median & 0-Cases \\
\hline Tasks & & & & \\
\hline Acquisition & 26 & 1,4 & 1,0 & 3 \\
\hline Social Interaction & 21 & 1,2 & 1,0 & 6 \\
\hline Apply expert knowledge & 18 & 1,0 & 1,0 & 5 \\
\hline Administration & 6 & 0,3 & 0,0 & 12 \\
\hline Requirements & & & & \\
\hline Soft Skills & 36 & 2,0 & 2,0 & 2 \\
\hline Proactivity & 25 & 1,4 & 1,0 & 2 \\
\hline Hard-Skills & 21 & 1,2 & 1,0 & 3 \\
\hline Efficiency & 14 & 0,8 & 1,0 & 8 \\
\hline Compensation & & & & \\
\hline Occupation & 32 & 1,8 & 2,0 & 4 \\
\hline Company & 32 & 1,8 & 2,0 & 5 \\
\hline Contract & 17 & 0,9 & 1,0 & 8 \\
\hline
\end{tabular}

As expected, the second reduction reveals a dominance of the acquisition components of the examined occupation in the area of Job Tasks. In the area of Job Requirements, there is a dominance of soft skills and descriptions of the high level of activity required. The reduction of the Job Compensation related codes resulted in a division into job specific, company specific and contract related contents. 


\section{Discussion and Conclusion}

The fact that occupational changers are addressed is only apparent from the titles of 2 companies. As a rule, this fact only becomes clear in the requirements. It is doubtful whether the target group addressed will read this paragraph if they do not find themselves in the title.

Similarly, in the case of the Job Tasks, although the description of the job as a whole is informative, the lack of information here is also rather remarkable. While there is general agreement on the task of acquiring new customers, only 7 of 18 companies state that insurances have to be sold and 4 that appointments have to be arranged with customers. Since these are the crucial components of the occupation in insurance sales, this considerable lack of information is likely to lead to a clear misconception of the candidates about the occupation. The second reduction emphasizes acquisition activities and social interaction. $2 / 3$ of the companies do not communicate any administrative activities.

In the area of Job Requirements, primarily social skills and practicableness are demanded. Although a cognitively challenging job can be assumed, only half of the companies explicitly require any completed vocational training. However, professional background in particular can have a significant influence on the probability of a successful occupational change into sales [13]. Also, according to [14], cognitive ability is one of the best predictors of occupational performance. The insurance companies examined in this study create the impression that a high level of motivation and social competence are sufficient for the job. Completely absent is the information about the legal requirements according to $\S 48$ (VAG), according to which insurance intermediaries have to meet their financial obligations reliably and live in orderly financial circumstances. This is in line with the results from [15] who found that frequently critical skills for workplace success are not demanded in online job advertisements.

In the section on Compensation, the Second Reduction is of particular interest. In terms of the ratio of average to median, the amount of information provided is more balanced than in the other areas. Also balanced and in line with the occupational changers target group, job-specific benefits are highlighted just as often as companyspecific ones. While the training program is highlighted by most companies, 8 companies do not provide any information on the employment contract. Only one company mentioned at least one specific salary component.
Overall, the content of information is low in all main categories. In particular, the tasks and compensations are not presented realistically, which can lead to a psychological contract breach and thus turnover [16]. Also [9] show a correlation between turnover and volume, relevance, depth and accuracy of the information received before starting the job. The question arises if communication, especially towards occupational changers, is sufficient in this form and if part of the traditionally high employee turnover in sales is also due to the fact that information is handled very sparingly even before the start of a career.

\section{References}

[1]. Lee, N., Sandfield, A., \& Dhaliwal, B. (2007). An empirical study of salesperson stereotypes amongst UK students and their implications for recruitment. Journal of Marketing Management, 23(78), 723-744.

[2]. Spence, M. (1973). Job Market Signaling. The Quarterly Journal of Economics, 87(3), 355-374.

[3]. Barber, A. E. (1998). Recruiting employees: Individual and organizational perspectives. Sage Publications.

[4]. Saks, A. M. (2017). The Im practicality of Recruitment Research. The Blackwell handbook of personnel selection, 47-72.

[5]. Wanous, J. P., Poland, T. D., Premack, S. L., \& Davis, K. S. (1992). The Effects of Met Expectations on Newcomer Attitudes and Behaviors: A Review and Meta-Analysis. Journal of Applied Psychology, 77(3), 288-297.

[6]. Breaugh, J. A., \& Starke, M. (2000). Research on employee recruitment: So many studies, so many remaining questions. Journal of management, 26(3), 405-434.

[7]. Bretz Jr, R. D., \& Judge, T. A. (1998). Realistic Job Previews: A Test of the Adverse Self-Selection Hypothesis. Journal of Applied Psychology, 83(2), 330-337.

[8]. Akerlof, G. A. (1978). The market for "lemons": Quality uncertainty and the market mechanism. In Uncertainty in economics (pp. 235-251). Academic Press.

[9]. Pitt, L. F., \& Ramaseshan, B. (1995). Realistic job information and salesforce turnover: an investigative study. Journal of Managerial Psychology, 10(5), 2936.

[10]. Earnest, D. R., Allen, D. G., \& Landis, R. S. (2011). Mechanisms linking realistic job previews with turnover: A meta-analytic path analysis. Personnel Psychology, 64(4), 865-897.

[11]. BaFin (2020). Erstversicherungsstatistik 2019, Bundesanstalt für Finanzdienstleistungsaufsicht, Bonn. Retrieved from:

https:/www.bafin.de/DE/PublikationenDaten/Statistik en/Erstversicherung/erstversicherung_artikel.html [accessed: 15 January 2021]. 
[12]. Mayring, P., \& Fenzl, T. (2014). Qualitative content analysis. In manual methods of empirical social research (pp. 543-556). Springer VS, Wiesbaden.

[13]. Cho, V., \& Ngai, E. W. (2003). Data mining for selection of insurance sales agents. Expert systems, 20(3), 123-132.

[14]. Van Iddekinge, C. H., Aguinis, H., Mackey, J. D., \& DeOrtentiis, P. S. (2018). A meta-analysis of the interactive, additive, and relative effects of cognitive ability and motivation on performance. Journal of Management, 44(1), 249-279.
[15]. Rios, J. A., Ling, G., Pugh, R., Becker, D., \& Bacall, A. (2020). Identifying critical 21 st-century skills for workplace success: A content analysis of job advertisements. Educational Researcher, 49(2), 8089.

[16]. Heffernan, M., \& Rochford, E. (2017). Psychological contract breach and turnover intention: the moderating effects of social status and local ties. The Irish Journal of Management, 36(2), 99-115. 